International Journal of Applied Mathematics

Volume 29 No. $4 \quad 2016,439-449$

ISSN: $1311-1728$ (printed version); ISSN: 1314-8060 (on-line version)

doi: http://dx.doi.org/10.12732/ijam.v29i4.3

\title{
A LAYER ANALYSIS OF PARAMETERIZED SINGULARLY PERTURBED BOUNDARY VALUE PROBLEMS
}

\author{
Mustafa Kudu $^{1 \S}$, Ilhame Amirali², Gabil M. Amiraliyev ${ }^{3}$ \\ ${ }^{1,3}$ Department of Mathematics \\ Faculty of Art and Sciences \\ Erzincan University \\ 24000, Erzincan, TURKEY \\ ${ }^{2}$ Department of Mathematics \\ Faculty of Art and Sciences \\ Duzce University \\ 81620, Duzce, TURKEY
}

\begin{abstract}
In this paper, the boundary value problems (BVP's) for parameterized singularly perturbed second order nonlinear ordinary differential equation are considered. The boundary layer behavior of the solution and its first and second derivatives have been established. Examples which are in agreement with the theoretical analysis are presented.
\end{abstract}

AMS Subject Classification: $34 \mathrm{~K} 10,34 \mathrm{~K} 26,34 \mathrm{~B} 08$

Key Words: dependence on a parameter, asymptotic bounds, singular perturbation, boundary layer

\section{Introduction}

We examine the asymptotic nature of solutions to the parameterized singularly perturbed second-order nonlinear boundary value problems with single boundary layer. An overview of some existence and uniqueness results and applications of parameterized equations may be obtained, for example, in [5-9, $14-16,18,20]$. In [1-3, 19] some approximating aspects of this kind of problems

$\begin{array}{ll}\text { Received: May 16, } 2016 & \text { (c) } 2016 \text { Academic Publications }\end{array}$

$\S$ Correspondence author 
have also been considered. The qualitative analysis of singular perturbation situations have always been far from trivial because of the boundary layer behavior of the solution. These problems are characterized by the presence of a small parameter that multiplies the highest order derivative, and they are stiff and there exists a boundary or interior layer where the solutions change rapidly $[4,10-13,17]$. In present note installed the uniform a priori estimates for the solutions of the problems (1)-(2) and (18)-(19) and their first and second derivatives. The obtained results are important for construction and analysis of appropriate approximate methods. Examples are presented to illustrate the theoretical results obtained.

\section{Asymptotic Estimates}

\subsection{Consider the Problem}

$$
\begin{gathered}
L u:=\varepsilon u^{\prime \prime}-f\left(t, u, u^{\prime}, \lambda\right)=0, \quad 0<t<T, \\
u(0)=\mu_{0}, u(T)=\mu_{1}, u^{\prime}(T)=\frac{\mu_{2}}{\varepsilon},
\end{gathered}
$$

where $0<\varepsilon<<1$ is the perturbation parameter, $\mu_{i}(i=0,1,2)$ are given constants and the function $f(t, u, v, \lambda)$ is assumed to be sufficiently continuously differentiable for our purpose function in $\{0 \leq t \leq T,-\infty<u, v, \lambda<\infty\}$ and

$$
0 \leq \frac{\partial f}{\partial u} \leq M_{1}^{*}, 0<\alpha \leq \frac{\partial f}{\partial v} \leq a *, 0<m_{1} \leq\left|\frac{\partial f}{\partial \lambda}\right| \leq M_{1}<\infty .
$$

By a solution of (1), (2) we mean the pair $\{u(t), \lambda\} \in C^{1}[0, T] \times \mathbb{R}$ for which problem (1), (2) is satisfied.

Theorem 1. For $\rho=1-\alpha^{-1} m_{1}^{-1} M_{1} M_{1}^{*} T>0$ and under conditions (3), the solution $\{u(t), \lambda\}$ of the problem (1) ,(2) satisfies

$$
|\lambda| \leq c_{0}
$$

and

$$
|u| \leq c_{1}
$$


where

$$
\begin{aligned}
& c_{0}=\rho^{-1}\left[\begin{array}{c}
\frac{m_{1}^{-1} a *\left(\left|\mu_{0}-\mu_{1}\right|+\alpha^{-1}\left|\mu_{2}\right|\right)}{T-(a *)^{-1}\left(1-e^{a * T}\right)}+m_{1}^{-1}\left(1+\alpha^{-1} M_{1}^{*}\right)\|F\|_{\infty} \\
+m_{1}^{-1} M_{1}^{*}\left(\left|\mu_{0}\right|+\left|\mu_{1}\right|\right)
\end{array}\right], \\
& c_{1}=\left|\mu_{0}\right|+\left|\mu_{1}\right|+\alpha^{-1} T\|F\|_{\infty}+\alpha^{-1} c_{0} M_{1} T
\end{aligned}
$$

$F(t)=f(t, 0,0)$ and

$$
\left|u^{(k)}(t)\right| \leq C\left(1+\frac{1}{\varepsilon^{k}} e^{-\frac{\alpha t}{\varepsilon}}\right), \quad k=1,2, t \in[0, T],
$$

provided $a \in C^{1}[0, T]$ and $\left|\frac{\partial f}{\partial t}\right| \leq C$ for $t \in[0, T]$ and

$|u| \leq c_{1},|v|<\infty,|\lambda| \leq c_{0}$.

Proof. We rewrite (1) in the form

$$
\varepsilon u^{\prime \prime}-a(t) u^{\prime}-b(t) u-\lambda c(t)-F(t)=0, \quad t \in[0, T],
$$

where $a(t)=\frac{\partial f}{\partial v}(t, \widetilde{u}, \widetilde{v}, \widetilde{\lambda}), b(t)=\frac{\partial f}{\partial u}(t, \widetilde{u}, \widetilde{v}, \widetilde{\lambda}), c(t)=\frac{\partial f}{\partial \lambda}(t, \widetilde{u}, \widetilde{v}, \widetilde{\lambda}), \widetilde{u}=\gamma u, \widetilde{v}=$ $\gamma v, \tilde{\lambda}=\gamma \lambda(0<\gamma<1)$-intermediate values. From (7) and (2) for the first derivative, we have

$$
\begin{aligned}
u^{\prime}(t) & =\frac{\mu_{2}}{\varepsilon} e^{-\frac{1}{\varepsilon} \int_{t}^{T} a(t) d t}-\frac{1}{\varepsilon} \int_{t}^{T} b(\tau) u(\tau) e^{-\frac{1}{\varepsilon} \int_{t}^{\tau} a(t) d t} d \tau \\
& -\frac{\lambda}{\varepsilon} \int_{t}^{T} c(\tau) e^{-\frac{1}{\varepsilon} \int_{t}^{\tau} a(t) d t} d \tau-\frac{1}{\varepsilon} \int_{t}^{T} F(\tau) e^{-\frac{1}{\varepsilon} \int_{t}^{\tau} a(t) d t} d \tau,
\end{aligned}
$$

Integrating this equality over $(0, T)$ we get,

$$
\begin{aligned}
\mu_{1}-\mu_{0} & =\frac{\mu_{2}}{\varepsilon} \int_{0}^{T} e^{-\frac{1}{\varepsilon} \int_{t}^{T} a(t) d t}-\frac{1}{\varepsilon} \int_{0}^{T}\left[\int_{t}^{T} b(\tau) u(\tau) e^{-\frac{1}{\varepsilon} \int_{t}^{\tau} a(t) d t} d \tau\right] d t \\
& -\frac{\lambda}{\varepsilon} \int_{0}^{T}\left[\int_{t}^{T} c(\tau) e^{-\frac{1}{\varepsilon} \int_{t}^{\tau} a(t) d t} d \tau\right] d t \\
& -\frac{1}{\varepsilon} \int_{0}^{T}\left[\int_{t}^{T} F(\tau) e^{-\frac{1}{\varepsilon} \int_{t}^{\tau} a(t) d t} d \tau\right] d t
\end{aligned}
$$


from which, we obtain

$$
\begin{aligned}
\lambda & =\frac{\varepsilon\left(\mu_{0}-\mu_{1}\right)}{\int_{0}^{T}\left[\int_{t}^{T} c(\tau) e^{-\frac{1}{\varepsilon} \int_{t}^{\tau} a(t) d t} d \tau\right] d t}+\frac{\mu_{2} \int_{0}^{T} e^{-\frac{1}{\varepsilon} \int_{t}^{T} a(t) d t} d t}{\int_{0}^{T}\left[\int_{t}^{T} c(\tau) e^{-\frac{1}{\varepsilon} \int_{t}^{\tau} a(t) d t} d \tau\right] d t} \\
& -\frac{\int_{0}^{T}\left[\int_{t}^{T} b(\tau) u(\tau) e^{-\frac{1}{\varepsilon} \int_{t}^{\tau} a(t) d t} d \tau\right] d t}{\int_{0}^{T}\left[\int_{t}^{T} c(\tau) e^{-\frac{1}{\varepsilon} \int_{t}^{\tau} a(t) d t} d \tau\right] d t}-\frac{\int_{0}^{T}\left[\int_{t}^{T} F(\tau) e^{-\frac{1}{\varepsilon} \int_{t}^{\tau} a(t) d t} d \tau\right] d t}{\int_{0}^{T}\left[\int_{t}^{T} c(\tau) e^{-\frac{1}{\varepsilon} \int_{t}^{\tau} a(t) d t} d \tau\right] d t} .
\end{aligned}
$$

Applying the mean value theorem for integrals, we deduce that,

$$
\begin{aligned}
\left|\frac{\int_{0}^{T}\left[\int_{t}^{T} F(\tau) e^{-\frac{1}{\varepsilon} \int_{t}^{\tau} a(t) d t} d \tau\right] d t}{\int_{0}^{T}\left[\int_{t}^{T} c(\tau) e^{-\frac{1}{\varepsilon} \int_{t}^{\tau} a(t) d t} d \tau\right] d t \mid}\right| & \leq \frac{\|F\|_{\infty}\left|\int_{0}^{T}\left[\int_{t}^{T} e^{-\frac{1}{\varepsilon} \int_{0}^{T} a(t) d t} d \tau\right] d t\right|}{m_{1} \int_{0}^{T}\left[\int_{t}^{T} e^{-\frac{1}{\varepsilon} \int_{0}^{T} a(t) d t} d \tau\right] d t} \\
& \leq m_{1}^{-1}\|F\|_{\infty}
\end{aligned}
$$

and

$$
\begin{aligned}
\left|\frac{\int_{0}^{T}\left[\int_{t}^{T} b(\tau) u(\tau) e^{-\frac{1}{\varepsilon} \int_{t}^{\tau} a(t) d t} d \tau\right] d t}{\int_{0}^{T}\left[\int_{t}^{T} c(\tau) e^{-\frac{1}{\varepsilon} \int_{t}^{\tau} a(t) d t} d \tau\right] d t}\right| \leq & \frac{M_{1}^{*}\|u\|_{\infty}\left|\int_{0}^{T}\left[\int_{t}^{T} e^{-\frac{1}{\varepsilon} \int_{0}^{T} a(t) d t} d \tau\right] d t\right|}{m_{1} \int_{0}^{T}\left[\int_{t}^{T} e^{-\frac{1}{\varepsilon} \int_{0}^{T} a(t) d t} d \tau\right] d t} \\
& \leq m_{1}^{-1} M_{1}^{*}\|u\|_{\infty} .
\end{aligned}
$$

Also, for first and second terms in right side of (9) for $\varepsilon \leq 1$ values, we have

$$
\begin{gathered}
\frac{\varepsilon\left|\mu_{0}-\mu_{1}\right|}{\left|\int_{0}^{T}\left[\int_{t}^{T} c(\tau) e^{-\frac{1}{\varepsilon} \int_{t}^{\tau} a(t) d t} d \tau\right] d t\right|}+\frac{\frac{\left|\mu_{2}\right|}{\varepsilon} e^{-\frac{1}{\varepsilon} \int_{0}^{T} a(t) d t}}{\left|\int_{0}^{T}\left[\int_{t}^{T} c(\tau) e^{-\frac{1}{\varepsilon} \int_{t}^{\tau} a(t) d t} d \tau\right] d t\right|} \\
\leq \frac{\varepsilon\left|\mu_{0}-\mu_{1}\right|}{m_{1}(a *)^{-1}\left[T-\varepsilon(a *)^{-1}\left(1-e^{-\frac{a * T}{\varepsilon}}\right)\right]}+\frac{\alpha^{-1} \varepsilon\left|\mu_{2}\right|\left(1-e^{-\frac{\alpha T}{\varepsilon}}\right)}{m_{1}(a *)^{-1}\left[T-\varepsilon(a *)^{-1}\left(1-e^{-\frac{a * T}{\varepsilon}}\right)\right]} \\
\leq \frac{m_{1}^{-1}\left(a *\left|\mu_{0}-\mu_{1}\right|+\alpha^{-1}\left|\mu_{2}\right|\right)}{T-(a *)^{-1}\left(1-e^{-a * T}\right)}
\end{gathered}
$$


It then follows from (10)-(12),

$$
|\lambda| \leq \frac{m_{1}^{-1}\left(a *\left|\mu_{0}-\mu_{1}\right|+\alpha^{-1}\left|\mu_{2}\right|\right)}{T-(a *)^{-1}\left(1-e^{-a * T}\right)}+m_{1}^{-1} M_{1}^{*}\|u\|_{\infty}+m_{1}^{-1}\|F\|_{\infty} .
$$

Next, we use the maximum principle for the differential operator $L v:=\varepsilon v^{\prime \prime}-$ $a(t) v^{\prime}-b(t) v, 0<t<T$ which is valid in the form: Suppose $v(t) \in C^{2}[0, T]$ be any function satisfying $L v \leq 0(0<t<T), v(0) \geq 0$ and $\quad v(T) \geq 0$. Then $v(t) \geq 0$ for all $t \in[0, T]$. Using the maximum principle with barrier functions

$$
\Psi_{ \pm}= \pm u(t)+\alpha^{-1} t\left(\|c\|_{\infty}+\|F\|_{\infty}\right)+\left|\mu_{0}\right|+\left|\mu_{1}\right|,
$$

we observe that

$$
\begin{gathered}
L \Psi_{ \pm}= \pm \lambda c(t) \pm F(t)-a(t) \alpha^{-1} t\left(\|c\|_{\infty}+\|F\|_{\infty}\right)-b(t)\left(\left|\mu_{0}\right|+\left|\mu_{1}\right|\right) \leq 0 \\
\Psi \pm(0) \geq 0, \quad \Psi^{\prime} \pm(T) \geq 0,
\end{gathered}
$$

therefore $\Psi \pm(t) \geq 0$, which yields

$$
\|u\|_{\infty} \leq\left|\mu_{0}\right|+\left|\mu_{1}\right|+\alpha^{-1} M_{1} T|\lambda|+\alpha^{-1} T\|F\|_{\infty} .
$$

Further from (7) by taking $\Phi(t)= \pm \lambda c(t)+F(t)$ we get

$$
\|u\|_{\infty} \leq\left|\mu_{0}\right|+\alpha^{-1}\left|\mu_{2}\right|+\alpha^{-1} T\|F\|_{\infty}+\alpha^{-1} M_{1} T|\lambda| .
$$

This inequality together with (13) immediately leads to (4), (5). After taking into consideration the uniformly boundedness in $\varepsilon$ of $u(t)$ and $\lambda$, it then follows from (8) that,

$$
\begin{aligned}
\left|u^{\prime}(t)\right| & \leq \frac{\left|\mu_{2}\right|}{\varepsilon} e^{-\frac{\alpha(T-t)}{\varepsilon}}+\frac{1}{\varepsilon} c_{1} M_{1}^{*} \int_{t}^{T} e^{-\frac{\alpha(\tau-t)}{\varepsilon}} d \tau \\
& +\frac{1}{\varepsilon} c_{0} M_{1} \int_{t}^{T} e^{-\frac{\alpha(\tau-t)}{\varepsilon}} d \tau+\frac{1}{\varepsilon}\|F\|_{\infty} \int_{t}^{T} e^{-\frac{\alpha(\tau-t)}{\varepsilon}} d \tau,
\end{aligned}
$$

which proves (6) for $k=1$. To obtain (6) for $k=2$, first from (1) we have

$$
\left|u^{\prime \prime}(T)\right| \leq \frac{F\left(T, u(T), u^{\prime}(T), \lambda\right)}{\varepsilon}
$$

from which after taking into consideration here $u^{\prime}(T)=\frac{\mu_{2}}{\varepsilon}$ and (4) we obtain

$$
\left|u^{\prime \prime}(T)\right| \leq \frac{C}{\varepsilon^{2}}
$$


Next, from differentiation (1) we have

$$
\begin{gathered}
\varepsilon w^{\prime \prime}-a(t) w^{\prime}-Q(t)=0, \quad 0<t<T, \\
w^{\prime}(T)=O\left(\varepsilon^{-2}\right)
\end{gathered}
$$

with

$$
\begin{gathered}
w(t)=u^{\prime}(t), \\
a(t)=\frac{\partial f}{\partial v}\left(t, u(t), u^{\prime}(t), \lambda\right), \\
Q(t)=\frac{\partial f}{\partial t}\left(t, u(t), u^{\prime}(t), \lambda\right)+\frac{\partial f}{\partial u}\left(t, u(t), u^{\prime}(t), \lambda\right) u^{\prime}(t)
\end{gathered}
$$

and due to our assumptions clearly,

$$
|Q(t)| \leq C\left(1+\frac{1}{\varepsilon} e^{-\frac{\alpha(T-t)}{\varepsilon}}\right) .
$$

Consequently, from (16), (17) we have

$$
\begin{gathered}
\left|w^{\prime}(t)\right| \leq \frac{C}{\varepsilon^{2}} e^{-\frac{\alpha(T-t)}{\varepsilon}}+\frac{1}{\varepsilon} \int_{t}^{T}|Q(t)| e^{-\frac{\alpha(T-t)}{\varepsilon}} \\
=\frac{C}{\varepsilon^{2}} e^{-\frac{\alpha(T-t)}{\varepsilon}}+C\left(1+\frac{1}{\varepsilon}\right) \int_{t}^{T} e^{-\frac{\alpha(T-t)}{\varepsilon}}
\end{gathered}
$$

which proves (6) for $k=2$.

Now consider the BVP:

$$
\begin{gathered}
L u:=\varepsilon u^{\prime \prime}+f\left(t, u, u^{\prime}, \lambda\right)=0, \quad 0<t<T, \\
u^{\prime}(0)=\frac{\mu_{0}}{\varepsilon}, u(T)=\mu_{1}, u^{\prime}(T)=\mu_{2},
\end{gathered}
$$

where $0<\varepsilon<<1$ is the perturbation parameter, $\mu_{i}(i=0,1,2)$ are given constants and the function $f(t, u, v, \lambda)$ is assumed to be sufficiently continuously differentiable for our purpose function in $\{0 \leq t \leq T,-\infty<u, v, \lambda<\infty\}$ and

$$
0 \leq-\frac{\partial f}{\partial u} \leq M_{1}^{*}, \quad 0<\alpha \leq \frac{\partial f}{\partial v} \leq a *, \quad 0<m_{1} \leq\left|\frac{\partial f}{\partial \lambda}\right| \leq M_{1}<\infty
$$


Theorem 2. For $\rho=1-\alpha^{-1} m_{1}^{-1} M_{1} M_{1}^{*} T>0$ and under conditions (20), the solution $\{u(t), \lambda\}$ of the problem (18), (19) satisfies

$$
\begin{aligned}
& |\lambda| \leq c_{0}, \\
& |u| \leq c_{1},
\end{aligned}
$$

where

$$
\begin{aligned}
& c_{0}=\rho^{-1}\left[\begin{array}{c}
\frac{a *\left|\mu_{2}\right|}{m_{1}\left(1-e^{a * T}\right)}+\frac{\alpha\left|\mu_{0}\right|}{m_{1}\left(e^{\alpha T}-1\right)}+m_{1}^{-1} M_{1}^{*}\left(\alpha^{-1}\left|\mu_{0}\right|+\left|\mu_{1}\right|\right) \\
+m_{1}^{-1}\left(1+\alpha^{-1} M_{1}^{*}\right)\|F\|_{\infty}
\end{array}\right], \\
& c_{1}=\alpha^{-1}\left|\mu_{0}\right|+\left|\mu_{1}\right|+\alpha^{-1} c_{0} M_{1} T+\alpha^{-1} T\|F\|_{\infty},
\end{aligned}
$$

$F(t)=f(t, 0,0)$ and

$$
\left|u^{(k)}(t)\right| \leq C\left(1+\frac{1}{\varepsilon^{k}} e^{-\frac{\alpha t}{\varepsilon}}\right), \quad k=1,2, t \in[0, T],
$$

provided $a \in C^{1}[0, T]$ and $\left|\frac{\partial f}{\partial t}\right| \leq C$ for $t \in[0, T]$ and $|u| \leq c_{1},|v|<\infty,|\lambda| \leq c_{0}$.

Proof. We rewrite (18) in the form

$$
\varepsilon u^{\prime \prime}+a(t) u^{\prime}-b(t) u-\lambda c(t)-F(t)=0, \quad t \in[0, T],
$$

where $a(t)=\frac{\partial f}{\partial v}(t, \widetilde{u}, \widetilde{v}, \widetilde{\lambda}), b(t)=-\frac{\partial f}{\partial u}(t, \widetilde{u}, \widetilde{v}, \widetilde{\lambda}), c(t)=-\frac{\partial f}{\partial \lambda}(t, \widetilde{u}, \widetilde{v}, \widetilde{\lambda}), \widetilde{u}=$ $\gamma u, \widetilde{v}=\gamma v, \widetilde{\lambda}=\gamma \lambda(0<\gamma<1)$-intermediate values. From (24) and (19) for the first derivative, we have

$$
\begin{aligned}
u^{\prime}(t) & =\frac{\mu_{0}}{\varepsilon} e^{-\frac{1}{\varepsilon} \int_{0}^{t} a(t) d t}+\frac{1}{\varepsilon} \int_{0}^{t} b(\tau) u(\tau) e^{-\frac{1}{\varepsilon} \int_{\tau}^{t} a(t) d t} d \tau \\
& +\frac{\lambda}{\varepsilon} \int_{0}^{t} c(\tau) e^{-\frac{1}{\varepsilon} \int_{\tau}^{t} a(t) d t} d \tau+\frac{1}{\varepsilon} \int_{t}^{T} F(\tau) e^{-\frac{1}{\varepsilon} \int_{\tau}^{t} a(t) d t} d \tau,
\end{aligned}
$$

from which, after using the initial condition $u^{\prime}(T)=\mu_{2}$ it follows that

$$
\begin{aligned}
\lambda & =\frac{-\mu_{2}}{\frac{1}{\varepsilon} \int_{0}^{T} c(\tau) e^{-\frac{1}{\varepsilon} \int_{0}^{\tau} a(t) d t} d \tau}+\frac{\frac{\mu_{0}}{\varepsilon} e^{-\frac{1}{\varepsilon} \int_{0}^{T} a(t) d t}}{\frac{1}{\varepsilon} \int_{0}^{T} c(\tau) e^{-\frac{1}{\varepsilon} \int_{0}^{\tau} a(t) d t} d \tau} \\
& -\frac{\frac{1}{\varepsilon} \int_{0}^{T} b(\tau) u(\tau) e^{-\frac{1}{\varepsilon} \int_{0}^{\tau} a(t) d t} d \tau}{\frac{1}{\varepsilon} \int_{0}^{T} c(\tau) e^{-\frac{1}{\varepsilon} \int_{0}^{\tau} a(t) d t} d \tau}-\frac{\frac{1}{\varepsilon} \int_{0}^{T} F(\tau) e^{-\frac{1}{\varepsilon} \int_{0}^{\tau} a(t) d t} d \tau}{\frac{1}{\varepsilon} \int_{0}^{T} c(\tau) e^{-\frac{1}{\varepsilon} \int_{0}^{\tau} a(t) d t} d \tau} .
\end{aligned}
$$


Thereby, analogously as in the Subsection 2.1, we arrive at

$$
|\lambda| \leq \frac{a *\left|\mu_{2}\right|}{m_{1}\left(1-e^{-a * T}\right)}+\frac{\alpha\left|\mu_{0}\right|}{m_{1}\left(e^{\alpha T}-1\right)}+m_{1}^{-1} M_{1}^{*}\|u\|_{\infty}+m_{1}^{-1}\|F\|_{\infty} .
$$

Next, we use the maximum principle for the differential operator $L v:=\varepsilon v^{\prime \prime}+$ $a(t) v^{\prime}-b(t) v, \quad 0<t<T$ which is valid in the form: Suppose $v(t) \in C^{2}[0, T]$ be any function satisfying $L v \leq 0(0<t<T), v^{\prime}(0) \leq 0$ and $v(T) \geq 0$. Then $v(t) \geq 0$ for any $0 \leq t \leq T$. Now, consider the barrier function

$$
\Psi_{ \pm}= \pm v(t)+\varepsilon\left|v^{\prime}(0)\right| \omega(t)+|v(T)| \alpha^{-1}(T-t)\|F\|_{\infty}
$$

where,

$$
\omega(t)=\alpha^{-1}\left(e^{\frac{-\alpha(T-t)}{\varepsilon}}-e^{\frac{-\alpha T}{\varepsilon}}\right) .
$$

Taking into consideration that, $\omega(t)$ is a solution of the problem

$$
\varepsilon \omega^{\prime \prime}+\alpha \omega^{\prime}=0, \omega^{\prime}(0)=-\frac{1}{\varepsilon}, \omega(T)=0,
$$

and it follows that

$$
\begin{gathered}
L \Psi_{ \pm}= \pm L v-a(t) \alpha^{-1}\|L v\|_{\infty}-b(t) \alpha^{-1} t\|L v\|_{\infty} \\
-b(t) \alpha^{-1}|v(T)|+\varepsilon\left|v^{\prime}(0)\right| L \omega(t) \\
\leq \varepsilon\left|v^{\prime}(0)\right| L \omega(t)\left(\varepsilon \omega^{\prime \prime}+a(t) \omega^{\prime}\right) \\
\leq \varepsilon\left|v^{\prime}(0)\right|(a(t)-\alpha) \omega^{\prime}(t)=\varepsilon\left|v^{\prime}(0)\right|(a(t)-\alpha)\left(-\frac{1}{\varepsilon} e^{\frac{-\alpha t}{\varepsilon}}\right) \leq 0, \\
\Psi_{ \pm}^{\prime}(0)= \pm v^{\prime}(0)+\varepsilon\left|v^{\prime}(0)\right|\left(-\frac{1}{\varepsilon} e^{\frac{-\alpha t}{\varepsilon}}\right)-\alpha^{-1}\|L v\|_{\infty} \\
\leq \pm v^{\prime}(0)+\varepsilon\left|v^{\prime}(0)\right| \leq 0, \\
\Psi^{\prime} \pm(T)= \pm v(T)+|v(T)| \geq 0 .
\end{gathered}
$$

therefore $\Psi \pm(t) \geq 0$, which leads to estimate

$$
\|v\|_{\infty} \leq \varepsilon \alpha^{-1}\left|v^{\prime}(0)\right|+|v(T)|+\alpha^{-1} T\|L V\|_{\infty} .
$$

Taking $v=u(t), L u=\lambda c(t)+F(t), u^{\prime}(0)=\frac{\mu_{0}}{\varepsilon}, u(T)=\mu_{1}, u^{\prime}(T)=\mu_{2}$ in (27) we obtain the following estimate for the solution of the problem (18),(19)

$$
\|u\|_{\infty} \leq \alpha^{-1}\left|\mu_{0}\right|+|\mu 1|+\alpha^{-1} M_{1} T|\lambda|+\alpha^{-1} T\|F\|_{\infty} .
$$

The inequalities (27), (28) immediately leads to (21), (22). Once the estimates (21), (22) are established, the estimate (23) easily follows from (24), analogously as in previous section. 
Here the validity of the obtained results is illustrated by two examples.

Example 3. Consider the following parameterized singular perturbation problem:

$$
\begin{gathered}
\varepsilon u^{\prime \prime}-2 u^{\prime}+\lambda+\tanh (1-t+\lambda)=f(t), \quad 0<t<1, \\
u(0)=0, u(1)=\mu_{1}, u^{\prime}(1)=\frac{\mu_{2}}{\varepsilon}
\end{gathered}
$$

with

$$
\mu_{1}=\frac{1+\varepsilon}{2+\varepsilon}, \mu_{2}=\frac{2 e+2+\varepsilon\left(1+e^{-\frac{2}{\varepsilon}}\right)}{1-e^{-\frac{2}{\varepsilon}}}
$$

and $f(t)$ selected so that the solution is

$$
u(t)=\gamma_{1}+\gamma_{2} e^{-\frac{2}{\varepsilon}(1-t)}+\frac{1}{2+\varepsilon} e^{1-t}, \lambda=0.5,
$$

where

$$
\gamma_{1}=-\frac{e+(1+\varepsilon) e^{-\frac{2}{\varepsilon}}}{(2+\varepsilon)\left(1-e^{-\frac{2}{\varepsilon}}\right)}, \gamma_{2}=\frac{1+e+\varepsilon}{(2+\varepsilon)\left(1-e^{-\frac{2}{\varepsilon}}\right)} .
$$

The first and second derivatives have the form

$$
u^{(k)}(t)=\left(\frac{2}{\varepsilon}\right)^{k} \gamma_{2} e^{-\frac{2}{\varepsilon}(1-t)}+\frac{1}{2+\varepsilon} e^{1-t}, k=1,2 .
$$

Therefore we observe here the accordance in our theoretical results described above.

Example 4. Consider the particular problem of type (18),(19) with

$$
\begin{gathered}
f\left(t, u, u^{\prime}, \lambda\right)=u^{\prime}-2(t+\varepsilon)+(2 \lambda+\cos \lambda \pi) e^{t}, \\
\mu_{0}=2, \mu_{1}=5-2 e^{-\frac{1}{\varepsilon}}, \mu_{2}=2\left(1+e^{-\frac{1}{\varepsilon}}\right) .
\end{gathered}
$$

The solution is given by

$$
u(t)=4+t^{2}-2 e^{-\frac{t}{\varepsilon}}, \quad \lambda=1 .
$$

The first and second derivatives have the form

$$
u^{(k)}(t)=2\left(t^{2-k}+(-1)^{k+1} \varepsilon^{-k} e^{-\frac{t}{\varepsilon}}\right), \quad k=1,2,
$$

which is in agreement with the theoretical bounds described above. 


\section{References}

[1] G.M. Amiraliyev, M. Kudu, H. Duru, Finite-difference method for parameterized singularly perturbed problem, J. Appl. Math., 3 (2004), 191-199.

[2] G.M. Amiraliyev, M. Kudu, H. Duru, Uniform difference method for a parameterized singular perturbation problem, Appl. Math. Comput., 175 (2006), 89-100.

[3] I.G. Amiraliyeva and G.M. Amiraliyev, Uniform difference method for parameterized singularly perturbed delay differential equations, Numer. Algor., 52 (2009), 509-521.

[4] P. A. Farrel, A.F. Hegarty, J.J.H. Miller, E. O'Riordan, G.I. Shishkin, Robust Computational Techniques for Boundary Layers, Chapman Hall/CRC, New York, 2000.

[5] M. Fěckan, Parametrized singularly perturbed boundary value problems, J. Math. Anal. Appl., 188 (1994), 426-435.

[6] I.A. Goma, Method of successive approximations in a two-point boundary problem with parameter, Ukrainian Math. J., 29 (1977), 594-599.

[7] T. Jankowski, and V. Lakshmikantham, Monotone iterations for differential equations with a parameter, J. Appl. Math. Stochastic Anal., 10 (1997), 273-278.

[8] T. Jankowski, Generalization of the method of quasilinearization for differential problems with a parameter, Dyn. Syst. Appl., 8 (1999), 53-72.

[9] T. Jankowski, Monotone iterations for differential problems, Math. Notes (Miskolc), 2 (2001), 31-38.

[10] M. Kudu, Asymptotic estimates for second-order parameterized singularly perturbed problem, Appl. Math., 5, No 13 (2014), 1988-1992.

[11] M. Kudu and I. Amirali, A priori estimates of solution of parametrized singularly perturbed problem, J. Appl. Math. Phys., 4, No 1 (2016), 7378.

[12] A.H. Nayfeh, Introduction to Perturbation Techniques, John Wiley \& Sons, New York, 1981. 
[13] Jr. R.E. O'Malley, Singular Perturbation Methods for Ordinary Differential Equations, Springer-Verlag, New York, 1991.

[14] T. Pomentale, A constructive theorem of existence and uniqueness for the problem, $y^{\prime}=f(x, y, \lambda), y(a)=\alpha, y(b)=\beta, Z$. Angew. Math. Mech., 56 (1976), 387-388.

[15] M. Rontó, and T. Csikos-Marinets, On the investigation of some nonlinear boundary value problems with parameters, Math. Notes (Miskolc) 1 (2000), 157-166.

[16] M. Rontó, On non-linear boundary value problems containing parameters, Arch. Math., 36 (2000), 585-593.

[17] H.G. Roos, M. Stynes and L. Tobiska, Robust Numerical Methods for Singularly Perturbed Differential Equations, Convection Diffusion and Flow Problems, Springer-Verlag, Berlin-Heidelberg, 2008.

[18] S. Staněk, Nonlinear boundary value problem for second order differential equations depending on a parameter, Math. Slovaca, 47 (1997), 439-449.

[19] M. Turkyilmazoglu, Analytic approximate solutions of parameterized unperturbed and singularly perturbed boundary value problems, Appl. Math. Model., 35 (2011), 3879-3886.

[20] P. Zhang, Existence of positive solutions for nonlocal second-order boundary value problem with variable parameter in Banach spaces, Fixed Point Theory Appl., 43 (2011), 1687-1812; doi:10.1186/1687-1812-2011-43. 
